\title{
Immunodetection of 5'-methylcytosine on Giemsa-stained chromosomes
}

\author{
Werner Pfarr ${ }^{1}$, Gerald Webersinke ${ }^{2}$, Christian Paar $^{1}$, and \\ Christian Wechselberger ${ }^{1}$ \\ ${ }^{1}$ Upper Austrian Research GmbH and ${ }^{2}$ Hospital of the Sisters of Mercy, Linz, Austria
}

BioTechniques 38:527-530 (April 2005)

In higher vertebrates, the major, if not the only, covalent modification of DNA is a methyl-group present at the $\mathrm{C} 5$ position of cytosine yielding $5^{\prime}$-methylcytosine (5'-MC). This modification occurs only in the context of $\mathrm{CpG}$ dinucleotides, short sequences that are significantly underrepresented in the genome but are found frequently concentrated within so called " $\mathrm{CpG}$ island" regions as well as in repetitive sequences (1). Several reasons might account for the statistical underrepresentation of $\mathrm{CpG}$ dinucleotides in the mammalian genome, one being that $5^{\prime}$-MC was shown to be a hotspot for mutation through deamination (2). Another aspect that has recently emerged addresses the issue of immunostimulation by unmethylated $\mathrm{CpG}$ sequences present in bacterial DNA, allowing the immune system to discriminate between sterile tissue damage and infectious danger (3). Previous studies on DNA methylation have already revealed a heterogeneous distribution of hypo- and hypermethylated areas on human metaphase chromosomes $(4,5)$. $\mathrm{CpG}$ islands are predominantly found at the $5^{\prime}$ ends of genes as well as in intronic regions, serving two major functions: (i) regulating the transcription levels of genes and (ii) protecting the host organisms against expression of undesired sequences (e.g., noncoding, repetitive, or parasitic ones) (6). Expressed genes are generally unmethylated, and aberrant methylation has been shown to be associated with many serious pathological consequences (7). For example, hypermethylation of tumor suppressor gene promoters is often associated with transcriptional down-regulation by interfering with the binding of, for example, transcription factors $(8,9)$. Several methods for obtaining methylation-patterns on a genomic scale are currently established, yet most of them are restricted to the analysis of purified, complex DNA preparations (6). On the other hand, immunodetection offers the possibility to obtain spatially resolved information on the distribution of 5'-MC on metaphase chromosomes $(10,11)$. We describe now an advanced experimental technique that renders the immunodetection of $5^{\prime}$-MC on human metaphase chromosome spreads previously stained by the Giemsa method possible. Due to the harsh conditions generally used during karyotype analysis, several modifications had to be introduced to maintain the chromosomal morphology during Giemsa staining, the destaining and DNA-denaturation procedures, and to facilitate the subsequent immunodetection of $5^{\prime}-\mathrm{MC}$ as outlined below.

Informed consent was obtained from a healthy volunteer, and venous blood was collected (Vacuette ${ }^{\circledR}$ system; Greiner Bio-One GmbH, Kremsmünster, Austria). Lymphocytes were purified by density gradient centrifugation (Ficoll-Paque ${ }^{\circledR}$; Amersham Biosciences Europe $\mathrm{GmbH}$, Vienna, Austria), and cells were cultured in PB-MAX ${ }^{\mathrm{TM}}$ karyotyping medium (Invitrogen $\mathrm{GmbH}$, Lofer, Austria) for 72 h at $37^{\circ} \mathrm{C}$. KaryoMAX ${ }^{\circledR}$ Colcemid $^{\circledR}$ solution (final concentration $0.2 \mu \mathrm{g} / \mathrm{mL}$; Invitrogen $\mathrm{GmbH}$ ) was added, and the cultures were incubated for an additional $1 \mathrm{~h}$. Cells were collected by centrifugation $(350 \times g$ for $8 \mathrm{~min}$ ) and resuspended in $0.1 \mathrm{~mL}$ culture media. For hypotonic treatment, $5 \mathrm{~mL} 0.047$ $\mathrm{M} \mathrm{KCl}$ were added dropwise, and the cell suspension was incubated for exactly $20 \mathrm{~min}$ at room temperature. After collecting the cells by centrifugation, the supernatant was discarded, and the cells were resuspended in $0.1 \mathrm{~mL}$ hypotonic solution. Five milliliters freshly prepared fixative (ice-cold methanol/acetic acid 3:1) were added dropwise, and the cell suspension was incubated for $5 \mathrm{~min}$ at room temperature. Cells were collected by centrifugation and resuspended in fresh fixative (repeated twice). The fixed cells can be stored at $-20^{\circ} \mathrm{C}$ for several months.

Slides were cleaned with $70 \%$ ethanol for $1 \mathrm{~min}$, rinsed in distilled water, and stored at $4^{\circ} \mathrm{C}$ until use. Cells were dropped on a cleaned slide, dried at $37^{\circ} \mathrm{C}$ on a moist filter paper, and incubated at $90^{\circ} \mathrm{C}$ for $30 \mathrm{~min}$. Subsequently, samples were treated for approximately $10 \mathrm{~s}$ with a $0.8 \%$ trypsin solution (BD Diagnostic Systems, Schwechat, Austria) at $37^{\circ} \mathrm{C}$. Giemsa staining was performed according to standard procedures (12). Samples were stained for 5 min, rinsed in water, and dried at $37^{\circ} \mathrm{C}$ for $15 \mathrm{~min}$. Images of metaphase chromosome spreads were acquired with an Axioplan 2 Imaging Microscope (Zeiss $\mathrm{GmbH}$, Vienna, Austria) and analyzed with the Power Gene MacKtype 5.7/ CytoVision 3.1 software (Applied Imaging International, Newcastle Upon Tyne, UK). Coordinates of selected chromosome spreads were recorded for subsequent interpretation of methylation data.

In order to ensure optimal preservation of the 5'-MC epitope for the following immunodetection procedure, destaining proved to be a very critical step and required several adaptations. After washing with phosphate-buffered saline (PBS) for $5 \mathrm{~min}$, the slides were dried for $30 \mathrm{~min}$. Postfixation with methanol/acetic acid (3:1) and additional destaining with methanol was performed for $5 \mathrm{~min}$ each, followed by air-drying for $60 \mathrm{~min}$. To produce accessible epitopes, DNA had to be denatured prior to the antibody incubation step. Samples were incubated in $2 \mathrm{M} \mathrm{HCl}$ for $30 \mathrm{~min}$ followed by neutralization with $0.1 \mathrm{M} \mathrm{NaBO}_{2}(\mathrm{pH} 8.5)$ for $5 \mathrm{~min}$ and air-drying for $60 \mathrm{~min}$. After the destaining procedure, slides were washed with Tris-buffered saline (TBS) for $5 \mathrm{~min}$, blocked with TBS/1\% casein overnight at $4^{\circ} \mathrm{C}$ and incubated with a monoclonal anti-5'-MC antibody (1:100 dilution; Serotec $\mathrm{GmbH}$, Düsseldorf, Germany) at $37^{\circ} \mathrm{C}$ for 60 min. Following a 5-min wash with TBS, samples were incubated with a 


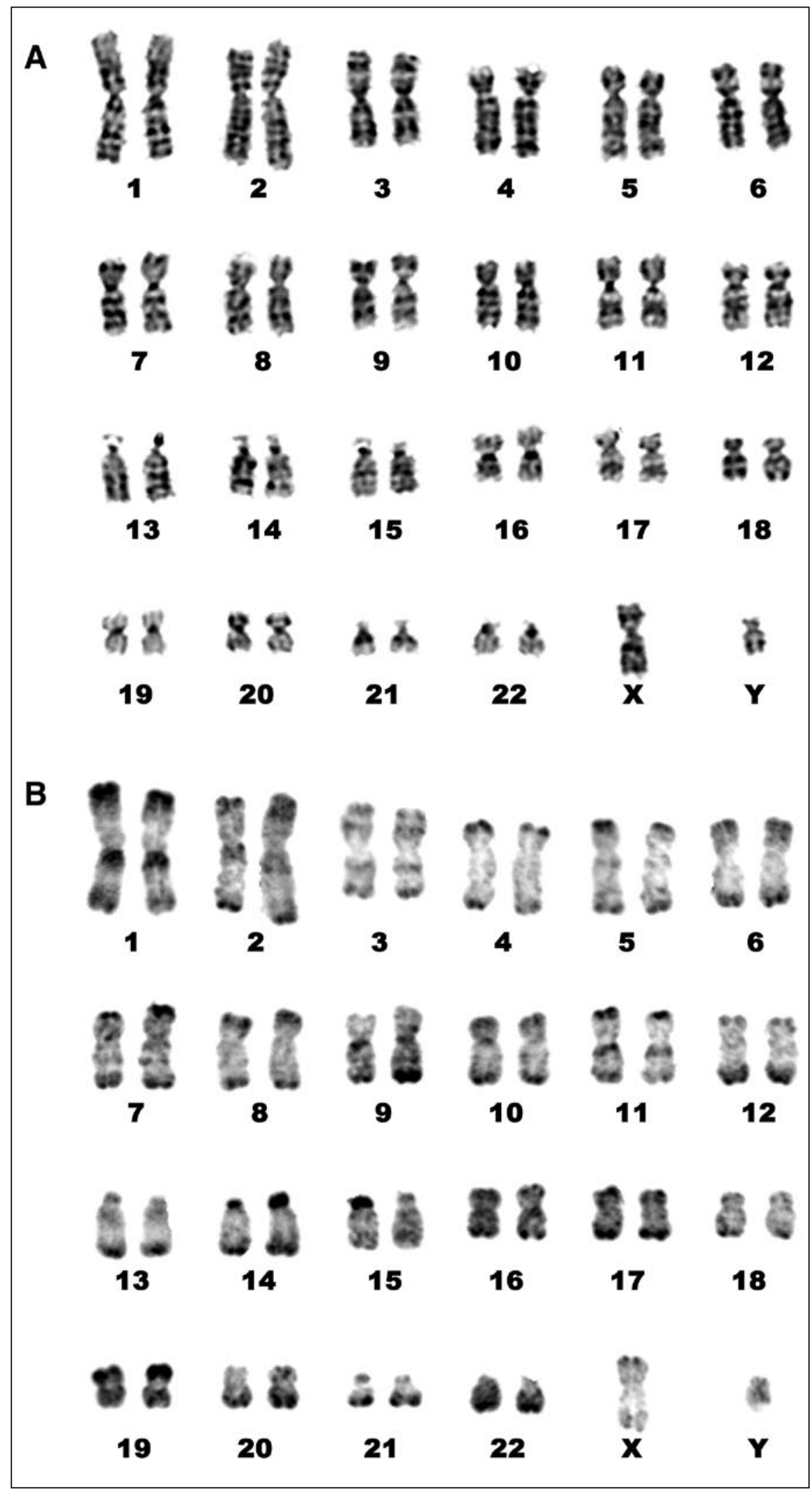

Figure 1. Metaphase chromosomes obtained from a normal human lymphocyte cell. (A) Karyogram of Giemsa-stained metaphase chromosomes. (B) Fluorescence image of the same chromosomes subsequently immunostained with a monoclonal antibody to detect $5^{\prime}$-methylcytosine.
Cy ${ }^{\mathrm{TM}} 3$-labeled secondary antibody (1:1000 dilution; Amersham Biosciences Europe $\mathrm{GmbH}$ ) at room temperature for $30 \mathrm{~min}$. After a brief wash with TBS, the slides were embedded with Universal Mount (Invitrogen $\mathrm{GmbH}$ ), and images were acquired using the CytoScout $^{\circledR}$ fluorescence microscope (Upper Austrian Research GmbH, Linz, Austria). Figure 1A shows a typical analysis of metaphase chromosomes stained by the Giemsa procedure. A fluorescence image of the same metaphase chromosomes after destaining and immunolabeling for the presence of $5^{\prime}$-MC according to our procedure is presented in Figure 1B. The potential of characterizing chromosomal changes in a lymphoma cell line is shown in Figure 2. Additional chromosomal material on chromosome 1 generated by a translocation $\mathrm{t}(1 ; 14)(\mathrm{q} 42 ; \mathrm{q} 32)$ could be identified by immunodetection of $5^{\prime}$-MC.

In conclusion, the procedure presented here is a simple yet efficient method for the immunodetection of $5^{\prime}$-MC on metaphase chromosomes. It can be used to obtain a fast and global overview of changes in genomic methylation patterns during the development of various types of cancer. Furthermore, the immunodetection of $5^{\prime}$-MC during the characterization of translocations might hold some promises for future applications, since local changes in DNA methylation have been already correlated with genome instability (13-15). The possibility to easily combine immunological fluorescence detection of hyper- and hypomethylated areas on metaphase chromosomes with standard Giemsa-staining procedures

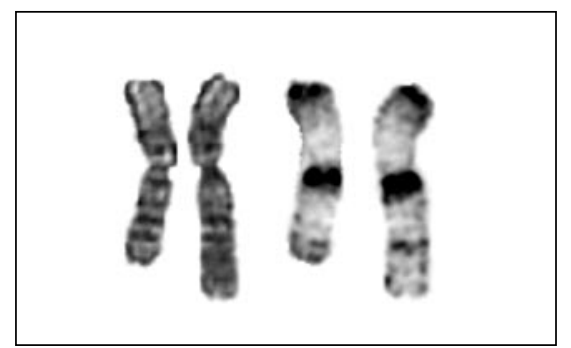

Figure 2. Characterization of translocated chromosomal material. Giemsa-stained metaphase chromosomes 1 from a human lymphoma cell line compared with a fluorescence image of the same chromosomes subsequently immunostained for $5^{\prime}$-methylcytosine. 
will greatly facilitate the characterization of possible disease-related methylation patterns on the genomic scale to eventually be used for diagnostic applications.

\section{ACKNOWLEDGMENTS}

This study was supported by grants from the Upper Austrian Government and from the Austrian Federal Ministry for Education, Science, and Culture (bm:bwk) in the context of the Gen-AU project "Ultra-Sensitive Proteomics and Genomics."

\section{COMPETING INTERESTS STATEMENT}

The authors declare no competing interests.

\section{REFERENCES}

1.Jones, P.A. and S.B. Baylin. 2002. The fundamental role of epigenetic events in cancer. Nat. Rev. Genet. 3:415-428.

2.Kow, Y.W. 2002. Repair of deaminated bases in DNA. Free Radic. Biol. Med. 33:886-893.

3.Stacey, K.J., G.R. Young, F. Clark, D.P. Sester, T.L. Roberts, S. Naik, M.J. Sweet, and D.A. Hume. 2003. The molecular basis for the lack of immunostimulatory activity of vertebrate DNA. J. Immunol. 170:3614-3620.

4.Sentis, C., P. Ludena, and J. FernandezPiqueras. 1993. Non-uniform distribution of methylatable CCGG sequences on human chromosomes as shown by in situ methylation. Chromosoma 102:267-271.

5. Bensaada, M., H. Kiefer, G. Tachdjian, J.M. Lapierre, V. Cacheux, A. Niveleau, and P. Metezeau. 1998. Altered patterns of DNA methylation on chromosomes from leukemia cell lines: identification of 5-methylcytosines by indirect immunodetection. Cancer Genet. Cytogenet. 103:101-109.

6.Havlis, J. and M. Trbusek. 2002. 5-Methylcytosine as a marker for the monitoring of DNA methylation. J. Chromatogr. B Analyt. Technol. Biomed. Life Sci. 781:373-392.

7.Robertson, K.D. and A.P. Wolffe. 2000. DNA methylation in health and disease. Nat. Rev. Genet. 1:11-19.

8.Herman, J.G. and S.B. Baylin. 2003. Gene silencing in cancer in association with promoter hypermethylation. N. Engl. J. Med. 349:2042-2054.

9.Wicki, R., C. Franz, F.A. Scholl, C.W. Heizmann, and B.W. Schafer. 1997. Repression of the candidate tumor suppressor gene $\mathrm{S} 100 \mathrm{~A} 2$ in breast cancer is mediated by site-specific hypermethylation. Cell Calcium 22:243-254.

10.Bernardino, J., M. Lombard, A. Niveleau, and B. Dutrillaux. 2000. Common methylation characteristics of sex chromosomes in somatic and germ cells from mouse, lemur and human. Chromosome Res. 8:513-525.

11.Bernardino-Sgherri, J. and B. Dutrillaux. 2001. Compaction, stainability and methylation of the late replicating $\mathrm{X}$ chromosome in mouse female fibroblasts. Cytogenet. Cell Genet. 94:79-81.

12.Drets, M.E. and M.W. Shaw. 1971. Specific banding patterns of human chromosomes. Proc. Natl. Acad. Sci. USA 68:2073-2077.

13.Nichol, K. and C.E. Pearson. 2002. CpG methylation modifies the genetic stability of cloned repeat sequences. Genome Res. 12:1246-1256.

14.Tuck-Muller, C.M., A. Narayan, F. Tsien, D.F. Smeets, J. Sawyer, E.S. Fiala, O.S. Sohn, and M. Ehrlich. 2000. DNA hy- pomethylation and unusual chromosome instability in cell lines from ICF syndrome patients. Cytogenet. Cell Genet. 89:121-128.

15.Schmutte, C. and R. Fishel. 1999. Genomic instability: first step to carcinogenesis. Anticancer Res. 19:4665-4696.

Received 23 November 2004; accepted 14 January 2005.

Address correspondence to Christian Wechselberger, Upper Austrian Research $\mathrm{GmbH}$, Center for Biomedical Nanotechnology, Scharitzerstr. 6-8, 4020 Linz, Austria. e-mail: christian.wechselberger@uar.at

\section{Shuttle system allowing simplified cloning of expression cassettes into advanced generation lentiviral vectors}

Ina Gruh, Kristin Schwanke, Stephanie Wunderlich, Ulrike Blömer, Michaela Scherr, Arnold Ganser, Axel Haverich, and Ulrich Martin

Leibniz Research Laboratories, Hannover, Germany

BioTechniques 38:530-534 (April 2005)

Recent lentiviral vector types have shown highly efficient stable transgene expression in terminally differentiated cells $(1,2)$. Despite major advantages of those vectors, lentiviral vector production and gene transfer remains labor-intensive and time-consuming and therefore not appropriate as the standard gene transfer approach for many laboratories.

Large vector size, resulting in low cloning efficiencies, and the absence of convenient multicloning sites leads to complex cloning strategies. In addition, during the cloning of full-length viral plasmid clones, homologous recombination between long terminal repeat (LTR) regions is frequently observed $(3,4)$ even in recA, recB, recJ, and $s b c C$ mutant bacterial strains and often results in the loss of the entire viral sequence except a single copy of an LTR (4).
Here we describe a novel lentiviral cloning system, LentiShuttle, which allows for fast and convenient generation of lentiviral vectors with different expression cassettes. The whole expression cassette is constructed within a high-copy plasmid with convenient multicloning sites. Finally, the cassette is transferred into the lentiviral vector via a simple restriction digest and standard sticky end ligation reaction. All cloning steps prior to the final transfer into pLentiShuttle, including those in pBShuttle, can be carried out in standard Escherichia coli strains such as TOP10 (Invitrogen, Karlsruhe, Germany), which can easily be prepared in the laboratory. Only for the very last cloning step, the insertion of complete expression cassettes between the viral LTRs of pLentiShuttle, is it recommended to use special (usually expensive) 